\title{
论文
}

\section{基于引导滤波和小波变换的太阳冕环结构 自动识别算法研究}

张礼 $^{1,2}$, 冯松 ${ }^{1,2 *}$

1. 昆明理工大学信息工程与自动化学院, 昆明 650500;

2. 昆明理工大学云南省计算机技术应用重点实验室, 昆明 650500

* E-mail: feng.song@kmust.edu.cn

收稿日期: 2018-03-07; 接受日期: 2018-07-30; 网络版发表日期: 2019-03-07

国家自然科学基金项目(批准号: 11463003, U1531132, 11573012)和云南省应用基础研究计划重点项目(编号: 2018FA035)资助

摘要冕环是太阳大气中的多热等离子在太阳磁场的作用下产生的一种太阳特征结构; 研究冕环动力学特征将 促进我们对太阳日冕磁场，日冕加热和日冕振荡等研究。冕环结构的准确识别和提取则是相关研究的前提条件. 然而由于日冕磁场的复杂性, 使得对冕环结构识别和提取面临很大的困境. 为此, 本文提出了一个新的冕环识别 算法. 该算法结合了引导滤波和小波变换模极大值的方法来对冕环结构进行自动识别和提取. 识别和提取算法过 程如下: (1) 运用模糊函数对太阳日冕图像的对比度进行增强; (2) 使用引导滤波对增强后的图像进行滤波来增强 冕环结构的边缘; (3) 对滤波过后的图像使用小波变换模极大值法来识别图像中的冕环结构, 并对识别出的冕环 图像进行二值化处理; (4) 对二值化图像进行形态学处理来获取冕环结构. 运用该算法对由过渡区和日冕探测器 (Transition and Coronal Explorer, TRACE)和太阳动力观测站的大气成像装置(Atmospheric Imaging Assembly on the Solar Dynamics Observatory, SDO/AIA)观测设备在171 凡波段所观测的日冕图像中的冕环结构进行识别和提 取, 并同已有文献的识别结果进行了对比, 结果表明本文所提出的算法具有很高的识别率和很好的鲁棒性. 所识 别和提取的冕环结构能进一步用于科学研究中.

关键词 小波变换,引导图像滤波, 模糊函数, 日冕冕环

\section{1 引言}

冕环结构是位于太阳日冕层中加热的等离子体沿 日冕磁场的磁力线流动形成的一种太阳特征结构 ${ }^{[1]}$, 它是日冕磁场结构的一种直观体现．研究表明太阳耀 斑、太阳风、日冕物质抛射、日冕振荡等很多太阳活 动都和日冕磁场存在着密不可分、息息相关的紧密联
系 ${ }^{[2,3]}$. 然而目前探测日冕磁场还存在一定的难度, 这 就使得太阳物理学家通过分析冕环的动力学特征来推 动对日冕磁场的探究, 进而促进日冕加热 ${ }^{[1,4,5]}$ 以及太 阳波动过程中的能量传递等重要科学问题的研究. 因 此, 对冕环结构进行准确的识别则是研究冕环结构的 动力学特征的前提.

目前已经有多种识别算法被提出. 识别方法主要

引用格式: 张礼, 冯松. 基于引导滤波和小波变换的太阳冕环结构自动识别算法研究. 中国科学: 技术科学, 2019, 49: 268-276

Zhang L, Feng S. Automatic detection of coronal loops using guided filtering and wavelet transform (in Chinese). Sci Sin Tech, 2019, 49: 268-276, doi: 10.1360/N092018-00046 
有以下 3 类: (1) 基于强度和梯度阈值 ${ }^{[6-9]}$; (2) 基于频域 (小波域)滤波 ${ }^{[10]}$; (3) 基于图像的相位一致性信息 ${ }^{[1,12]}$. 我们对冕环的成因及其图像特征进行分析后发现：首 先，冕环结构的各处等离子体由于受太阳磁场的影响 不同而分布不均匀，造成冕环结构本身强度较大以及 它们自身的边缘梯度较小导致冕环结构边缘模糊不 清; 其次, 在日冕图像中, 某些相邻冕环之间相距很近 且它们的边缘梯度差异很小, 导致在对其进行识别时 很难将它们区分开来; 最后, 由于冕环出现的地方通 常都伴随着其他太阳活动的发生，如耀斑、海绵状亮 斑(Solar Moss)等, 当这些太阳活动出现时, 会对冕环 识别的准确性产生干扰 ${ }^{[1]}$. 这使得目前在对冕环结构 进行自动提取时往往存在如下主要问题: (1) 检测到 的冕环结构完整性较差; (2) 检测到的冕环数量较少; (3) 将某些非冕环结构检测为冕环结构, 造成误检.

McAteer等人 ${ }^{[10]}$ 提出了运用小波变换模极大值方 法(Wavelet Transform Modulus Maxima, WTMM)来识 别和提取冕环结构. 该方法是使用一个二维平滑函数 的偏导数作为小波函数, 然后对图像进行多尺度小波 变换, 获取其局部模极大值, 得到识别的冕环结构; 该 方法在冕环的识别和提取中可以检测到大多数冕环, 且各个冕环的连接性也比较好. 但是该方法对那些较 弱的冕环结构却不能够很好地检测出来, 而且还会将 其他非冕环结构检测为冕环. 见文献[10]图1.

为了克服目前现有技术的局限性，本文提出了一 种基于引导滤波和小波变换的冕环特征结构自动识别 算法. 首先对原始的日冕图像进行对比度增强,使得较 暗的冕环强度与背景强度的比值得到增强, 这样做是 为了在之后的冕环检测中能够检测到细小较暗的环结 构; 然后使用引导滤波来抑制噪声和增强冕环结构的 边缘特征; 最后，我们使用小波变换模极大值法来提 取图像中的冕环边缘结构.

\section{2 冕环检测}

首先简要介绍引导滤波技术, 然后详细介绍冕环 的识别过程.

\section{1 引导滤波}

引导滤波是 $\mathrm{He}$ 等人 ${ }^{[13]}$ 在 2010 年提出的一种新型 滤波器, 它能在对图像噪声进行平滑的同时来感知并
增强图像特征边缘. 目前已被广泛应用于图像去雾、 边缘增强、图像复原等领域. 引导滤波是通过一个局 部线性模型, 运用引导图像来对输入图像进行滤波操 作以此来平滑输入图像的同时去增强图像特征的边 缘. 引导图像可以是输入图像本身也可以是另外一幅 不同于输入图像的其他图像.

引导滤波的关键是在引导图像 $\boldsymbol{I}$ 和滤波输出图像 $\boldsymbol{q}$ 之间构建一个局部线性模型, 其用公式表示如下:

$q_{i}=a_{k} I_{i}+b_{k}, \forall i \in w_{k}$,

其中, $I_{\mathrm{i}}$ 代表引导图像在窗口 $w_{k}$ 中的第 $i$ 个像素点. $a_{k}$ 和 $b_{k}$ 是该线性方程在窗口 $w_{k}$ 中的线性常数，其中 $k$ 表示引 导图像中的第 $k$ 个像素. 对方程(1)求导可以得到输出 图像的梯度信息, 即 $\nabla q=a \nabla I$ 这确保了只有当引导 图像 I中存在特征边缘时, 滤波输出图像 $\boldsymbol{q}$ 中才会出现 特征边缘.

由于输出图像是输入图像去掉噪声后的结果, 在 对图像使用引导滤波时, 想要的结果是在尽可能去除 噪声的同时保留特征边缘信息, 也就是要使得输出图 像与输入图像的差异尽可能的小. 因此, 为了寻求一 种方法, 使其既能使得输出图像和输入图像差异最小 同时还能使得输出图像和引导图像满足方程(1)所示 的线性模型，文献[13]在窗口 $w_{k}$ 中建立了一个关于输 出图像与输入图像的局部代价函数

$$
E\left(a_{k}, b_{k}\right)=\sum_{i \in w_{k}}\left(\left(a_{k} I_{i}+b_{k}-p_{i}\right)^{2}+\epsilon a_{k}^{2}\right),
$$

其中, $\epsilon$ 是一个正则化参数, 用来防止 $a_{k}$ 的取值过大, 由 方程(1)可知, $a_{k} I_{i}+b_{k}-p_{i}$ 表示输出图像 $\boldsymbol{q}$ 与输入图像 $\boldsymbol{p}$ 在窗口 $w_{k}$ 中对应的第 $i$ 个像素点之间的差值. 为了使得 方程(2)式取得最小值，采用文献[14]介绍的线性回归 方法求解方程(2), 求得局部线性模型中的线性常系数 $a_{k}$ 和 $b_{k}$ 分别为

$a_{k}=\frac{\frac{1}{|w|} \sum_{i \in w_{k}} I_{i} p_{i}-\mu_{k} \overline{p_{k}}}{\sigma_{k}^{2}+\epsilon}$,

$b_{k}=\overline{p_{k}}-a_{k} \mu_{k}$,

式中, $u_{k}$ 和 $\sigma_{k}^{2}$ 分别表示引导图像在局部窗口 $w_{k}$ 中的均 值和方差, $|w|$ 表示窗口 $w_{k}$ 内的像素点个数, $\bar{p}$ 是输入 图像 $\mathrm{p}$ 在窗口内的均值.

当线性常数 $a_{k}$ 和 $b_{k}$ 确定后，通过方程(1)就可以求 
得滤波输出 $\boldsymbol{q}$.

在本文中, 由于是要对图像中的特征边缘进行保 留, 因此输入图像与引导图像是同一幅图像. 即当 $\boldsymbol{p}=\boldsymbol{I}$ 时, 方程式(3)可改写为 $a_{k}=\frac{\sigma_{k}^{2}}{\sigma_{k}^{2}+\epsilon}$, 相应的方程式(4)可 改写为 $b_{k}=\mu_{k}-a_{k} \mu_{k}$. 当 $\epsilon=0$ 时, $a_{k}=1, b_{k}=0$, 此时可以得 到滤波输出的值与引导图像在该点的取值是一样的; 当 $\epsilon>0$ 时, 我们考虑以下两种情况: (1) 如果图像在窗口 $w_{k}$ 中的像素变化较大时, 可以得到 $\sigma_{k}^{2} \gg \epsilon$, 由方 程 $a_{k}=\frac{\sigma_{k}^{2}}{\sigma_{k}^{2}+\epsilon}$ 和 $b_{k}=\mu_{k}-a_{k} \mu_{k}$ 可得 $a_{k} \approx 1, b_{k} \approx 0 ; \quad$ 此时 判定其为边缘像素并将其保留. (2) 如果图像在窗口 $w_{k}$ 中的像素变化基本不变时, 可以得到 $\sigma_{k}^{2} \ll \epsilon$, 此时, 可 得 $a_{k} \approx 0, b_{k} \approx 1$; 此时判定其为非边缘像素并将其 平滑.

\section{2 冕环的检测过程}

为了描述和展示所提的算法的识别过程，本文选 用了由文献 $[6 \sim 8,10,15]$ 所使用观测数据，它们是由过
渡区和日冕探测器(Transition and Coronal Explorer, TRACE)在1998年5月19日22:21 UT 由 $171 \AA$ 波段的成 像数据, 其大小是 $1024 \times 1024$ 像素, 如图1(a)所示. 选用 该数据的目的是为了和已有文献的识别结果来对比, 以此来评价所提算法的性能.

步骤一: 图像归一化. 本文首先使用图像的最大值 和最小值来对图像的强度进行归一化, 把原始图像强 度归一化到 $[0,1]$ 区间; 读入图像记为 $\boldsymbol{I}_{\mathrm{or}}$, 归一化后的 图像记为 $\boldsymbol{I}_{\mathrm{out}}$, 则其具体公式为

$I_{\text {out }}=\frac{\left(I_{\text {or }}-\min \left(I_{\text {or }}\right)\right)}{\left(\max \left(I_{\text {or }}\right)-\min \left(I_{\text {or }}\right)\right)}$.

步骤二: 图像对比度增强. 由于等离子体的密度不 均匀使得冕环结构本身的强度分布不均匀, 而且一些 冕环结构特征不明显，如图1(a)中使用矩形框C和D所 指出的那些区域的冕环结构. 为此, 我们使用文献[16] 中所采用的模糊增强算法来对图像进行模糊对比度增 强. 该算法具有增强图像对比度的能力. 在这里, 我们 使用该方法增大了隶属度矩阵中大于 0.35 的元素的值, 同时, 减小了隶属度矩阵中小于等于 0.35 的元素的值, 最后得到的图像如图1(b)所示. 通过图1(a)和(b)的对比
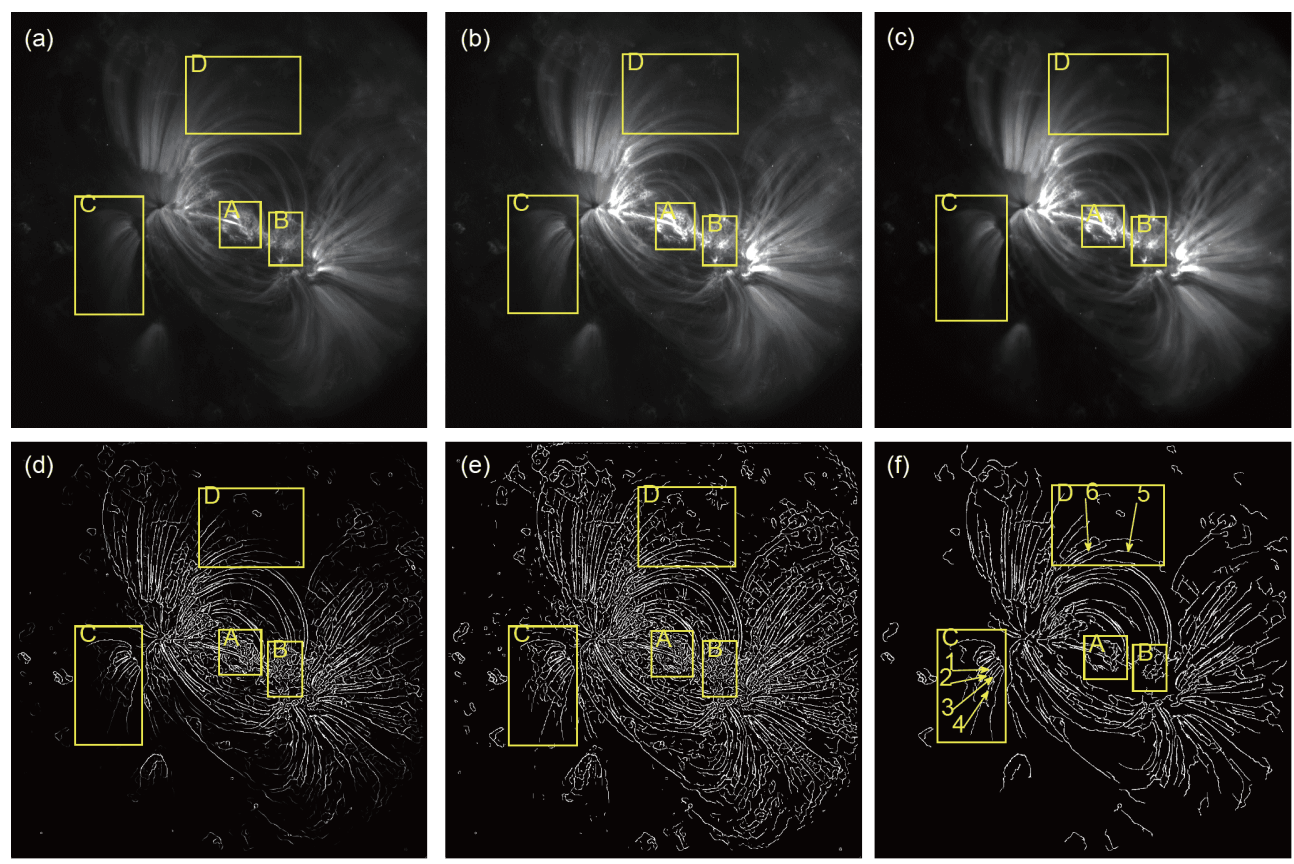

图 1 (网络版彩图)冕环识别过程. (a) 1998年5月19日22:21 UT 由171 波段的成像数据, 图像尺寸为 $1024 \times 1024$. 为了描述和 对比我们标记了 4 个区域, 其中矩形框A和B标记solar moss特征, 矩形框C和D标记较暗的冕环结构. (b) 对(a)进行模糊增强后的 图像; (c) 对(b)进行引导滤波后的图像; (d) 小波变换模极大值后的图像; (e) (d) 二值化后的图像所选择的阈值为 $0 \sim 0.7$; (f) 形态 学处理后的图像, 其中箭头所指的为识别出的较暗冕环 
发现，经过模糊对比度增强后的日冕图像中冕环结构 更加清晰，较弱的冕环结构得到了一定的增强，如矩 形框 $\mathrm{C}$ 和 $\mathrm{D}$ 所指示的区域.

步骤三: 冕环结构边缘增强. 对图1(b)观察可以发 现，在冕环结构得到增强的同时非冕环的结构也得到 了增强，如图1(a)中方框A和B的区域中的moss结构, 同时图像中冕环结构的边缘仍然比较模糊. 为此, 本 文使用引导滤波来增强冕环结构的边缘同时平滑噪 声. 在本文中我们把 $\epsilon$ 的值设置为 $\epsilon=0.0005$, 并选择了 一个 $5 \times 5$ 的窗口; 引导滤波后得到的结果如图 1 (c) 所示. 结果表明，使用引导滤波后的冕环结构边缘得到了很 好的增强.

步骤四: 小波变换模极大值. 通常, 使用小波变换 模极大值来检测边缘时，一般在不同尺度下采用一个 二阶可导平滑函数来对图像进行平滑, 然后由该函数 一阶微分的极大值或二阶微分的零交叉点来判定突变 点. 本文中我们选择二维高斯函数作为平滑函数, 即取 平滑函数 $\theta(x, y)=\frac{1}{2 \pi \sigma^{2}} \mathrm{e}^{-\frac{x^{2}+y^{2}}{2 \sigma^{2}}}$, 其中本算法中 $\sigma$ 取值为 0.64 , 令 $\theta_{S}(x, y)=\frac{1}{S^{2}} \theta\left(\frac{x}{S}, \frac{y}{S}\right)$, 其中 $S$ 表示小波变换的尺 度, 平滑函数 $\theta_{S}(x, y)$ 分别对 $x, y$ 求二阶偏导数, 得两小 波函数分别为 $\varphi_{S}^{1}(x, y)=\frac{\partial^{2}(x, y)}{\partial x^{2}}, \varphi_{S}^{2}(x, y)=\frac{\partial^{2}(x, y)}{\partial y^{2}}$, 接 着采用这两个小波函数来对图像进行小波变换，选择 滤波器长度 $N=200$, 得到图像在局部区域的模极大值. 由于图像中存在着非冕环结构, 这就使得通过小波变 换后得到的模极大值点并非全为冕环边缘像素点, 为 了能够有效地去除伪边缘，本文分别对在各尺度下得 到的模极大值点进行阈值处理. 此处的阈值用 thr 1 表 示, 在这里将阈值 $\mathrm{thr} 1$ 设置为 0.00405 (关于选取不同的 阈值对冕环识别的影响将在后面进行讨论). 之后运用 边缘点梯度方向的特征进一步去除得到图像中的伪边 缘. 最终得到的图像如图1(d)所示. 从图1(d)可以看出, 这些模极大值点很好地显示出了图像中冕环的边缘 结构.

步骤五: 图像二值化. 为了能够提取出冕环边缘, 对图1(d)采用阈值来二值化图像. 在这里选取的二值 化阈值为 $\mathrm{thr}$ 2小于 0.7 (二值化阈值的选取对实验结果 的影响在后面将进行讨论), 其处理结果如图1(e)所示.

步骤六: 形态学处理. 为了消除二值图像中的非冕 环结构边缘，通过图1(e)对冕环边缘和非冕环边缘的 分析, 发现非冕环边缘都相对较短, 可以对图1(e)进行 形态学处理来去除小于一定面积的连通对象. 在本文 中, 我们设定面积为 80 像素, 得到的图像如图1(f)所示.

通过以上步骤, 最终得到了识别出的冕环结构, 如 图1(f)所示. 从图1(f)可以看出, 本文算法对冕环结构有 很高的识别率能够识别出包括箭头标记出的细小较暗 冕环结构在内的大部分冕环结构, 并且识别出的冕环 结构完整性也比较好.

\section{3 分析与讨论}

\section{1 同已有算法识别结果的对比}

为了验证本文所提方法的准确性, 我们将本文图1 (f)与文献[10]中的图1以及文献[15]中的图5(a)人工绘 制的冕环边缘结构进行比较, 发现本文所提的方法识 别出的冕环边缘相比文献[10]所识别出的冕环边缘效 果更好.

为了更清晰地对比识别结果，本文在图中选择两 个包含较弱冕环的区域, 如图1(a)中的矩形框 C和D所 示. 为了能够比较直观地看到本文识别的结果与文献 [10]中识别结果的差异, 将区域C和D放大后如图2(a) 和(b)所示, 同时它们的识别结果如图2(c)和(d)所示. 从图2(a)和(b)中可以很清楚地看到, 图中箭头所指的


图 2 (网络版彩图)(a)和(b)分别表示图1(a)中区域C和D放 大后的图像; (c) 和(d)分别表示区域C和D识别出的冕环结构 
地方冕环是真实存在的，我们的识别结果也可以通过 文献[15]中的图5(a)人工绘制的冕环边缘图像来得到 证实. 从文献[10]中的图1相应的区域可以看到，这些 冕环没有被检测到或者检测的不完整，而通过图2(c) 和(d)可以看到，图2(a)和(b)中箭头所指的冕环都被很 好地检测出来, 由此可知本文所提的方法可以很好地 检测到这些较暗的冕环.

由以上的对比结果可以看出，本文所用的方法检 测到的冕环边缘结构相比文献[10]中图1检测到的冕 环边缘结构更加完整, 检测到的数目更多, 更接近文献 [15]中图5(a)人工绘制的冕环结构.

当然，通过将本文图1(f)与文献[15]中图5(a)进行 比较可以看到, 虽然本文中已经使用了形态学处理来 去除小面积连通域，但是图1(f)仍然存在着一些非冕 环结构. 为此，在这里本文对这些非冕环结构进行对 比和分析. 图1(a)为原始图像，其中矩形框 $\mathrm{A}$ 和 $\mathrm{B}$ 中为 海绵状亮斑; 图3(a)为本文识别出的冕环结构图, 其中 矩形框A和B是与图1(a)相对应的区域; 将本文图3(a) 框出的区域与文献[15]中图5(a)人工绘制的冕环结构 相应的区域进行比较, 发现框中为非冕环结构. 通过对 图1(a)中框出的两个区域的海绵状亮斑进行分析可知, 这些海绵状亮斑在图中由于其亮度比相当一部分冕环 结构的亮度都大, 因此, 在选取小的阈值把较暗的冕环 结构识别出来的同时，也就相应的把其他结构也识别 出来. 又由于这些海绵状亮斑所占的区域比较大，且 和冕环结构交织在一起，这就使得本文采用的形态学 去除小面积连通域不能有效地将其去除. 为了能够有 效去除这些非冕环结构，本文对非冕环结构的形态进 行分析，可以看到图3(a)中框出的非冕环结构的像素 点在较小的区域内分布比较密集, 而冕环结构的像素 点或者在横轴上分布较长或者在坚轴上分布较长，这 就使得非冕环结构的像素点在图像二维矩阵中的横坚 轴方向的索引值的方差都比较小; 而冕环结构的像素 点在图像矩阵中或者横轴或者坚轴或者横坚轴方向上 的索引值的方差较大. 因此, 本文首先采用形态学中的 方法来计算连通分量, 得到标记矩阵和连通分量的总 数; 其次计算标记矩阵中各连通分量的 $x, y$ 坐标, 并分 别计算各连通分量中所有像素点的 $x$ 坐标和 $y$ 坐标的方 差; 然后分别将两个方差与一定大小的值进行比较, 当 两个方差都小于给定值时，将该连通分量设置为非冕 环结构, 否则为冕环结构; 最后, 在图中去除这些判定
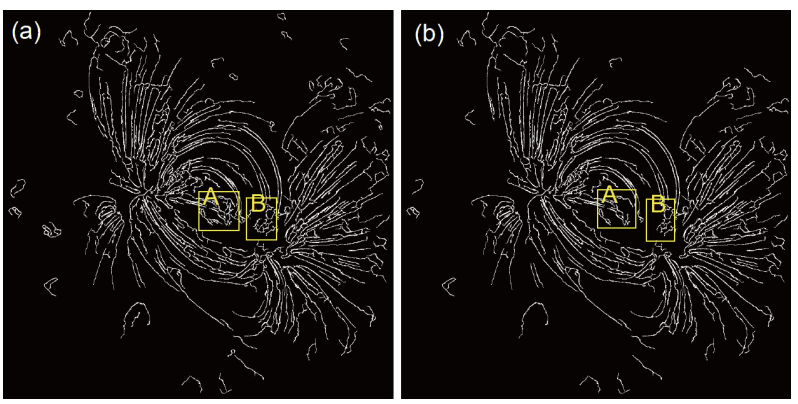

图 3 (网络版彩图)(a) 本文的识别结果, 矩形框A, B中为识 别出的非冕环结构; (b) 去除部分非冕环结构后的图像

为非冕环结构的连通分量, 得到最后的识别结果. 其结 果如图3(b)所示, 通过将图3(b)中矩形框框出的区域与 图3(a)相应区域进行比较可以发现，图3(a)中识别出来 的非冕环结构被部分去除掉. 由此, 证明该方法可以在 一定程度上去除这些非冕环结构.

\section{2 阈值讨论}

在算法中，我们使用两个阈值来对图像进行处理， 两个阈值分别用thr1，thr2来代表. 为了验证不同阈值 对冕环的检测所产生影响, 接下来首先对阈值thr1进 行验证，对阈值 thr 1 分别选取 2 个间值 $(0.00105$, 0.02005)在各尺度上对图像进行去噪, 最终得到的图 像分别如图4(a)和(b)所示.

通过图4(a)和(b)与图1(d)进行比较可以得出, 阈值 thr1的大小对检测到的冕环的数量和完整性都有影响: 当阈值较小时，会得到许多非冕环结构; 当阈值较大 时，会使得检测到的冕环不够完整且数量减少. 实验 结果表明, 对于不同的图像阈值 $\operatorname{th} 1$ 的选取和图像自 身的像素值分布有关，在使用时应结合具体的特征提 取目标来调整该阈值.

接下来我们进一步来讨论阈值 $\mathrm{thr} 2$ 对冕环边缘识 别的影响, 在此选择 $\mathrm{thr} 1=0.00405$, 对阈值thr2分别选取 两个区间 $0.006<\mathrm{thr} 2<0.7$ 和 $0.01<\mathrm{thr} 2<0.7$, 得到的实验 结果如图2(c)和(d)所示.

将图1(e)与图4中(c)和(d)两图进行对比可以很清 楚地看到, 当阈值thr 2 小于 0.7 时, 检测到的冕环在数量 和完整性上都要好于阈值thr2在区间0.006 0.7和在区 间0.01 0.7 时检测到的冕环，由此可得当阈值thr2选择 较小范围时，会使得二值化后的图像中冕环的数量较 少且完整性较差; 反之, 当阈值 $\mathrm{thr} 2$ 选择较大的范围 时, 虽然在二值化后的图像中冕环结构数量较多且完 

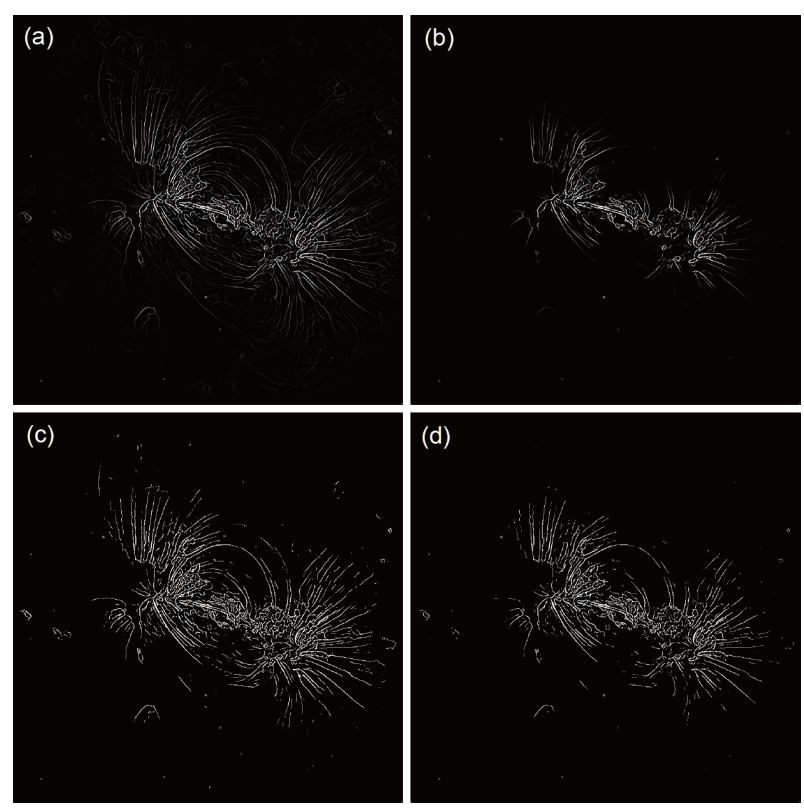

图 4 (a)和(b)分别表示阈值 $\operatorname{thr} 1$ 取(0.00105, 0.02005)时得到 的结果; (c)和(d)分别表示阈值thr2在区间0.006 0.7 和在区间 $0.01 \sim 0.7$ 时得到的结果

整性也较好, 但是, 也会将更多的非冕环结构保留在图 像中.

\section{3 适用性讨论}

为了验证所提算法的适用性, 本文又选取了 3 组由
不同观测设备在 $171 \AA$ 波段所获取的数据. 它们是由 TRACE在1999年11月06日由 $171 \AA$ 波段在日面边缘所 观测到的冕环结构, 其大小是 $768 \times 768$ 像素(如图 $5(\mathrm{a})$ 所 示); 由太阳动力观测站的大气成像装置(Atmospheric Imaging Assembly on the Solar Dynamics Observatory, SDO/AIA)在2011年2月14日23:36 UT在171 ̊波段观 测到的数据, 其大小是 $723 \times 723$ 像素(如图 5 (b)所示); 由SDO/AIA于2011年9月6日22:17 UT在 $171 \AA$ 波段观 测到的数据, 其大小是 $834 \times 835$ 像素(如图 5 (c) 所示). 将 本文的算法分别应用到上面3组数据, 识别结果分别显 示在图5(d)，(e)和(f)中. 因为每幅图像大小各不相同, 所以我们在设定阈值thr1, thr2和面积去噪时, 对图 5 (a), (b) 和 (c)选择的 thr 1 , thr2和面积 $S 1$ 的值分别为 (thr1 $=0.00205,0.003<$ thr $2<0.7, S 1=80),(\operatorname{thr} 1=0.00085$, $0.0015<$ thr $2<0.7, S I=80)$ 和 $($ thr $1=0.00105,0.001<$ thr $2<$ $0.7, S 1=80)$.

由图5(a), (b)和(c)三幅图像的冕环边缘检测结果 图可以看出，本文算法可以检测到图像中的大部分冕 环结构边缘, 并且检测出的冕环边缘的完整性也比 较好.

然而我们也注意到，通过原始图像与结果图像进 行对比发现: (1) 本文的方法对相互交叉的冕环结构 的识别还存在一定的局限性，尤其在相交点的附近检 测出的冕环边缘存在断裂的情况；(2) 当冕环的附近
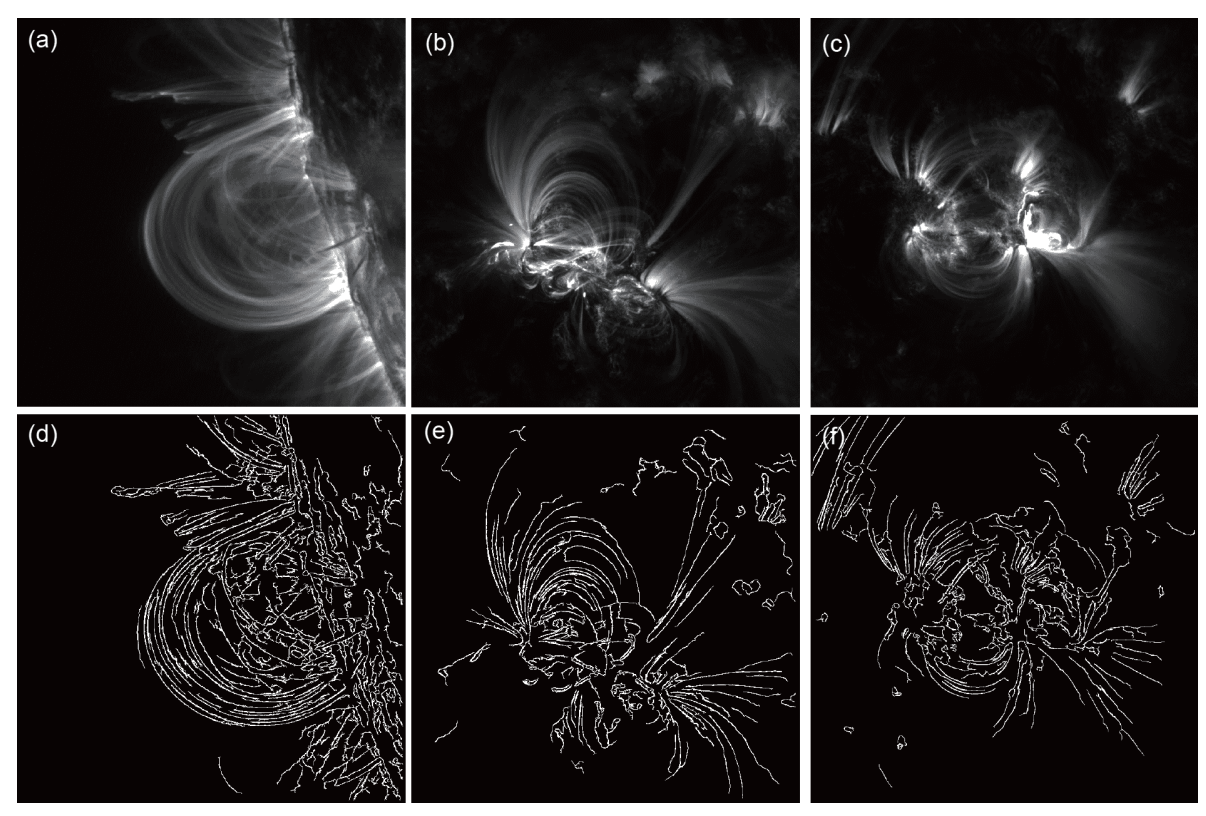

图 5 图(a) ( c) 为原始图像, 图(d) (f)为图(a) ( c) 的识别结果 
出现其他非冕环的太阳活动并且这些太阳活动在图像 中的强度比冕环强度大或者相近且活动区域较大时, 该方法不能很好地检测到这些冕环, 使得最后的结果 图像中这些非冕环结构仍然被保留了下来, 如图5(f) 所示.

通过以上的分析可以得到, 虽然本文的方法在检 测冕环边缘时存在着以上这些不足, 但它仍然可以很 好地检测到图像中的大部分冕环. 由此可知, 本文的 方法适用性比较好, 对于不同时间, 不同种类的冕环 图像都能够比较好的检测出冕环的边缘结构.

\section{4 引导滤波技术讨论}

由文献[13]可知, 引导滤波在进行滤波时能够较 好地保留边缘等细节; 由于引导滤波通过比较所选窗 口中所有像素值的方差与正则化参数的大小来判断该 窗口中的中心像素点是属于“平坦”区域还是“高方差” 区域. 即, 如果图像中一个像素处在“高方差”区域的中 间, 那么在引导滤波后它的值是不变的, 而如果图像中 一个像素处在“平坦”区域的中间, 那么在引导滤波后 它的值是窗口 $w_{k}$ 中其他像素的平均值. 而从前面对冕 环特征进行的分析可知, 在冕环结构的足点附近通常 伴随着非冕环结构, 且它们之间的强度差异较小, 而 在环顶的冕环结构较暗, 使得它与周围的区分不太明 显, 因此正则化参数也就不能太大, 因为过大的正则 化参数会使得引导滤波将部分“高方差”区域误判为 “平坦”区域, 当然也不能太小, 因为太小会使得引导滤 波将“平坦”区域误判为“高方差”区域.

引导滤波可以增强冕环边缘结构与背景像素间的 对比度, 使得本来较为模糊的边缘变的较为清晰, 同 时, 使得相邻冕环结构能够得到更好地区分, 使得后 面能够更为准确地识别冕环结构.

在太阳观测图像中, 从图像的形态和边缘特征方 面也存在着和冕环相类似的图像特征, 如色球纤维和 太阳针状体. 为此本文也对含有该类特征的太阳图像 进行了边缘特征的增强实验. 图6(a)为由中国云南天 文台一米新真空太阳望远镜在 $\mathrm{H} \alpha$ 波段观测的图像中 的色球纤维结构(图6(a))以及由SOT/EIT在日面边缘所 观测的针状体图像(图6(c))进行了引导滤波, 图6(b)和 (d)为增强后的图像. 而通过对比图6(a), (c)和(b), (d), 图6(b)中的纤维结构和(d)中的针状体都分别得到了显 著增强. 由此证明引导滤波是可以用在类似的边缘特
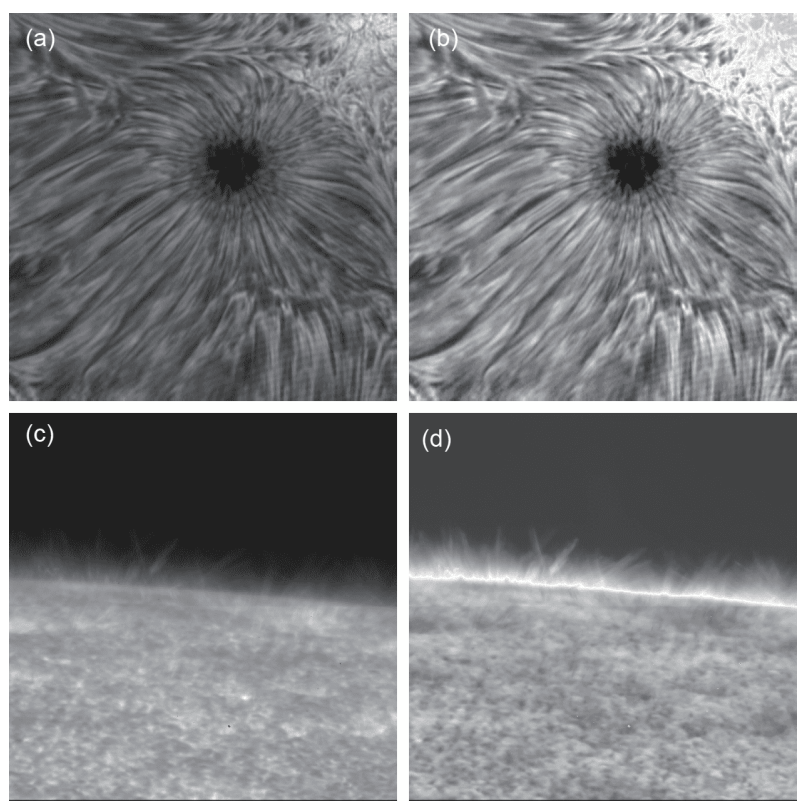

(d)

图 6 (a)和(b)分别表示太阳色球纤维原始图像和太阳色球 纤维增强后的图像; (c)和(d)分别表示太阳针状体原始图像 和太阳针状体增强后的图像

征的梯度较小, 边缘特征不明显的特征结构上, 如太 阳色球纤维和太阳针状体的图像增强中.

\section{4 总结}

由于太阳图像中的冕环结构本身从冕环足点到环 顶逐渐减弱, 相邻冕环间的强度变换小, 以及在冕环出 现的附近通常还伴随着其他太阳活动等这些复杂情况 造成了冕环结构的检测与提取比较困难. 在过去的几 十年中，许多检测冕环结构的算法被提出来，并且这 些算法都能够比较有效地检测到冕环结构, 但是它们 也有各自的不足, 它们检测到的冕环在冕环结构的完 整性, 清晰程度和数量上都不是特别好.

本文针对文献[10]的不足, 在使用WTMM方法之 前, 对冕环图像进行了预处理, 主要通过对原始图像 进行模糊对比度增强处理, 增强原始图像中强度较暗 的冕环结构, 之后运用引导滤波保留边缘的特性将它 运用到增强后的图像中, 使得冕环结构的边缘得到保 留的同时对非冕环区域的像素采用局部均值代替. 这 样可以有效地消除其他非冕环太阳活动的干扰. 在完 成了对图像的相关预处理后, 最后使用小波变换模极 大值法得到日冕图像中的冕环结构. 由本文的实验可 
以看出，本文得到的冕环结构不论完整性还是检测到 的冕环数量都比文献[10]中检测到的冕环结构好，对 那些比较暗的冕环结构也可以很好地检测到. 当然, 我们从本文检测到的冕环结构图中可以看出，有部分 非冕环结构, 如solar moss等由于其与冕环结构交织在
了一起, 且它们之间的强度也比较相近, 使得很难将 它们分离开来而被留在了图中, 从图中可以看出仍有 一些冕环没有检测出来而且对于相交的冕环结构检 测效果较差. 这也是我们接下来需要着手去解决的 问题.

\section{参考文献}

1 Schmelz J T. Are coronal loops isothermal? Astrophys J, 2002, 578: L161-L164

2 Durak N, Nasraoui O, Schmelz J. Coronal loop detection from solar images. Pattern Recognition, 2009, 42: 2481-2491

3 Vaiana G S, Rosner R. Recent advances in coronal physics. Annu Rev Astron Astrophys, 1978, 16: 393-428

4 Schmelz J T, Scopes R T, Cirtain J W. Determining coronal heating of plasma loops through differential emission measure analysis. Adv Space Res, 2002, 30: 507-516

5 Schmelz J T, Cirtain J W, Beene J E, et al. Coronal loops: Isothermal OR multithermal? Adv Space Res, 2003, 32: 1109-1115

6 Aschwanden M J, Lee J K, Gary G A, et al. Comparison of five numerical codes for automated tracing of coronal loops. Sol Phys, 2008, 248: 359-377

7 Lee J K, Newman T S, Gary G A. Oriented connectivity-based method for segmenting solar loops. Pattern Recognition, 2006, 39: 246-259

8 Lee N J K, Newman T S, Gary G A. Dynamic aperture-based solar loop segmentation. In: Proceedings of the 7th IEEE SSIAI. IEEE Computer Society, 2006. 91-94

9 Steger C. An unbiased detector of curvilinear structures. IEEE Trans Pattern Anal Mach Intell, 2002, 20: 113-125

10 McAteer R T J, Kestener P, Arneodo A, et al. Automated detection of coronal loops using a wavelet transform modulus maxima method. Sol Phys, 2010, 262: 387-397

11 李洪波, 赵明宇, 刘显. 基于相位一致性的冕环识别方法. 天文学报, 2016, 57: 402-421

12 冯松, 王洋, 赵燕, 等. 基于相位一致性和方向滤波冕环震荡识别与跟踪. 天文研究与技术, 2016, 13: 345-350

13 He K, Sun J, Tang X. Guided Image Filtering. Comput Vis ECCV 2010. Berlin, Heidelberg: Springer, 2010. 1-14

14 Draper N R, Smith H. Applied regression analysis. 2nd ed. New York: John Wiley \& Sons, 1981

15 Lee J K, Tang W K. Snake-based technique for automated coronal loop segmentation. In: Proceedings of the 19th International Conference in Central Europe on Computer Graphics, Visualization and Computer Vision in cooperation with EUROGRAPHICS Association. Plzen, 2011. 3340

16 Pal S K, King R A. Image enhancement using fuzzy set. Electron Lett, 1980, 16: 376-378 


\title{
Automatic detection of coronal loops using guided filtering and wavelet transform
}

\author{
ZHANG $\mathrm{Li}^{1,2}$ \& FENG Song ${ }^{1,2}$ \\ ${ }^{1}$ Faculty of Information Engineering \& Automation, Kunming University of Science \& Technology, Kunming 650500, China; \\ ${ }^{2}$ Yunnan Key Laboratory of Computer Technology Applications, Kunming University of Science \& Technology, Kunming 650500, China
}

The corona is the outer layer of the solar asmosphere. The bright loop-like structures in the corona, which are called coronal loops, are hot plasma to be bound to the solar coronal magnetic fields. Studying these magnetic features can help to better understand the dynamics of coronal magnetic fields and coronal oscillations, and further clearify the debate of coronal heating. Accurate identifying coronal loops are crucial for the relevant studies. However, due to the complexity of the coronal magnetic fields, it is very difficult to identify and extract the loop-like structures. In this case, we propose a novel algorithm based on guided filtering and wavelet transform modulus maxima to automatically detect and extract them. The steps are as follows: we use (1) a fuzzy function to enhance the contrast of solar corona images; (2) guided filtering to highlight the edges of coronal loops; (3) wavelet transform modulus maxima to identitify the edges of coronal loops; (4) image binarization to extract coronal loops; (5) morphological operate to remove those non-loop structures. The images observed by the Transition and Coronal Explorer (TRACE) and the Atmospheric Imaging Assembly on the Solar Dynamics (SDO/AIA) intruments in the $171 \AA$ channel are selected to illustrate the porcess and further evaluate the performance of the algorithm. The results demonstrate that the proposed algorithm has significant advantages over provious identification methods and the detected and extracted structures can be further applied to scientific researchs.

wavelet transform, guided image filtering, fuzzy function, coronal loop

doi: 10.1360/N092018-00046 